\title{
Introduction to the special section on hepatocellular carcinoma treatment response
}

\author{
Mishal Mendiratta-Lala ${ }^{1} \cdot$ Vahid Yaghmai ${ }^{2}$
}

Published online: 13 July 2021

(c) The Author(s), under exclusive licence to Springer Science+Business Media, LLC, part of Springer Nature 2021

Hepatocellular carcinoma (HCC) is the sixth most common tumor worldwide and third leading cause of cancer-related death. The majority of patients with HCC have two competing disease processes, which include the cancer itself and the underlying chronic liver disease. The optimal curative treatment for HCC is transplant; however, long wait times can result in progression of HCC. Thus, there are a host of liver-directed therapies which can be considered for patients awaiting transplantation in order to bridge therapy to reduce progression and possible post-transplant tumor recurrence. Similarly, liver-directed therapies can help downstage patients to meet the criteria to become eligible for transplantation. The most common liver-directed therapies include thermal ablation (radiofrequency and microwave ablation), transarterial chemoembolization, transarterial bland embolization, transarterial radioembolization, and stereotactic body radiotherapy. In addition, there are a host of new systemic and immunologic treatment options. The landscape of HCC treatment has changed and has become significantly more complex.

Imaging assessment after locoregional therapy is critical to guide management decisions in clinical care, including transplant evaluation. There are multiple image-based treatment response assessment classification systems and criteria which have undergone modification over time to improve response assessment. However, in order to accurately assess the treatment response, it is necessary for the radiologist to understand not only the different types of treatment options and their mechanism of action, but also the expected treatment-specific imaging findings of each form of locoregional therapy. Furthermore, due to the complex nature of these

Mishal Mendiratta-Lala

mmendira@med.umich.edu

Vahid Yaghmai

vyaghmai@hs.uci.edu

1 University of Michigan, Ann Arbor, USA

2 University of California Irvine, Irvine, USA patients' imaging, treatment history, and chronic liver disease, management of these patients is nearly always via a multidisciplinary effort.

In this special edition, the role of diagnostic imaging and interventional radiology in the treatment of $\mathrm{HCC}$ is explored, including a summary of the different treatment options, expected imaging findings after locoregional therapy, expected imaging after combined therapies, and areas of local and distant recurrence, including metastatic patterns of spread. Furthermore, the role of multidisciplinary tumor board evaluation, future of HCC therapy, and emerging and advanced imaging techniques are described, such as contrast-enhanced ultrasound and radiomics of HCC treatment response.

It is an honor and privilege to serve as guest editors. We would like to thank the Society of Abdominal Radiology (SAR) for the opportunity to create the HCC Treatment Response Disease Focused Panel (DFP) as a new platform in 2020. This effort was made possible with the knowledge, time, and expertise of all the members of the SAR DFP and the contributing authors. We have made every effort to provide a broad review of HCC treatment response imaging by providing contributions of experts from a wide range of different academic institutions and specialties. We are grateful to Dr. Daniel Johnson, the Editor-in-Chief of Abdominal Radiology, for this wonderful opportunity, and for his guidance throughout the process. We hope that this special issue on HCC treatment response provides a valuable reference to general radiologists as well as radiologists specializing in hepatic treatment response imaging and management.

Mishal Mendiratta-Lala MD Vahid Yaghmai MD

Publisher's Note Springer Nature remains neutral with regard to jurisdictional claims in published maps and institutional affiliations. 